\title{
Imaging techniques in transcatheter aortic valve replacement
}

This article was published in the following Dove Press journal:

Research Reports in Clinical Cardiology

21 November 2013

Number of times this article has been viewed

\author{
Robert A Quaife \\ Jennifer Dorosz \\ John C Messenger \\ Ernesto E Salcedo \\ Division of Cardiology, University \\ of Colorado, Aurora, CO, USA
}

Correspondence: Ernesto E Salcedo Division of Cardiology, University of Colorado, Mail Stop BI20, 12605 East 16th Avenue, Aurora, CO 800045 , USA Tel +l 7208487565

Fax +| $720848530 \mid$

Email ernesto.e.salcedo@ucdenver.edu
Abstract: Calcific aortic stenosis is now understood as a complex valvular degenerative process sharing many risk factors with atherosclerosis. Once patients develop symptomatic calcific aortic stenosis, the only effective treatment is aortic valve replacement. In the past decade, transcatheter aortic valve replacement (TAVR) has been developed as an alternative to surgery to treat severe calcific aortic stenosis. Cardiac imaging plays a pivotal role in the contemporary management of patients with calcific aortic stenosis, and particularly in patients being considered for TAVR, who demand detailed imaging of the aortic valve apparatus. In this review, we highlight the role of cardiac imaging for patient selection, procedural guidance, and evaluation of results of TAVR.

Keywords: aortic stenosis, cardiovascular imaging, transcutaneous aortic valve replacement

\section{Introduction}

Calcific aortic stenosis (AS), once thought to be a normal consequence of aging, is now understood as a complex valvular degenerative process sharing many risk factors with atherosclerosis. ${ }^{1}$ Currently, there is no known treatment to prevent its development, no effective means to halt its progression, and no accepted pharmacologic regimen to decrease its severity; once patients develop severe symptomatic calcific AS, the only effective treatment is aortic valve replacement. ${ }^{2,3}$ Until recently, surgical aortic valve replacement was the only viable option for these patients. In the past decade, transcatheter aortic valve replacement (TAVR) has been developed as an alternative to surgery to treat severe calcific AS. ${ }^{4,5}$ This procedure is currently applicable in patients with severe trileaflet AS who are either not surgical candidates or have an exceedingly high surgical risk. With a prevalence of $3.4 \%$, the burden of disease in the elderly due to severe AS is substantial. In North America, under current indications, approximately 100,000 elderly patients with severe AS are TAVR candidates, and about 10,000 patients become eligible for TAVR annually. ${ }^{6}$ Already, this relatively new technique has been used in over 40 countries, accumulating to over 50,000 implantations. ${ }^{7}$ TAVR has been shown to compare favorably with conventional surgical aortic valve replacement. ${ }^{8}$ As experience is gained with this new technology, the applications for this procedure are likely to expand, eg, there is a recent report demonstrating the feasibility of TAVR in patients with bicuspid aortic valve. ${ }^{9}$ The initial results of TAVR have been very encouraging and have generated a great deal of interest and enthusiasm among physicians taking care of patients with AS. ${ }^{10}$ Furthermore, TAVR has resulted in marked improvements in health status and quality of life compared with standard therapy over 1 year of follow-up. ${ }^{11}$ 
Paradoxical low-flow low-gradient AS, despite normal left ventricular (LV) ejection fraction, is a recently described entity that has been shown to be associated with a more advanced stage of the disease and worse prognosis. Dobutamine stress echo (DSE) greatly aids risk stratification and clinical decision-making in these patients. Valve calcium quantification by multidetector computed tomography (CT) and measurement of plasma brain natriuretic peptide may also be helpful for the management of patients with no LV flow reserve in whom DSE is often inconclusive. TAVR may eventually prove to be an attractive alternative to surgical AVR in both types of low-flow low-gradient severe AS, but this remains to be confirmed by future randomized studies. ${ }^{12}$

Successful management of patients with severe AS and particularly patients being considered for TAVR demands detailed imaging of the aortic valve apparatus. Cardiac imaging plays a pivotal role in patient selection, procedure guidance, and evaluation of results. ${ }^{13}$ In this review, we present advances in cardiovascular imaging as they relate to calcific AS and TAVR.

\section{Aortic valve complex}

The introduction of TAVR has renewed interest in the aortic valve complex..$^{14,15}$ Recent review articles have emphasized the presence of a virtual annulus at the nadir of the leaflets, a coronet-shaped annulus following the insertion of the leaflets into the sinuses of Valsalva, and an annulus at the sinotubular junction ${ }^{16}$ (Figure 1). Successful TAVR deployment depends on precise measurements of these structures. Because of a limited number of prosthesis sizes (20, 23, 26, and $29 \mathrm{~mm}$ [Edwards Lifesciences, Irvine CA, USA] and 23, 26, 29, and $31 \mathrm{~mm}$ [CoreValve; Medtronic, Minneapolis, MN, USA]), choosing the correct one depends on accurate and precise measurements of the virtual annular size and the sinotubular junction dimensions. Choosing a prosthesis that is too small for the annular size creates the potential for periprosthetic leak or prosthesis embolization; choosing a prosthesis too large for the annulus creates the potential for annular disruption.

TAVR is currently performed only in patients with trileaflet AS, therefore analyzing the number of leaflets is crucial for patient selection. In patients with normal aortic leaflets, this is a relatively easy endeavor; however, in patients with heavily calcified leaflets and severe commissure fusion, this can be more difficult to ascertain. The three aortic cusps are named after the sinus of Valsalva, where they are situated, the right coronary cusp being situated in the sinus of Valsalva with the right coronary ostium, the left coronary cusp on the left coronary sinus of Valsalva, and the noncoronary cusp on the remaining sinus of Valsalva. In addition to housing the coronary ostia, the sinus of Valsalva provides space for the leaflets to expand and open fully. In the presence of a significant amount of calcification on the annulus or leaflets, the sinuses of Valsalva provide additional room for the calcium

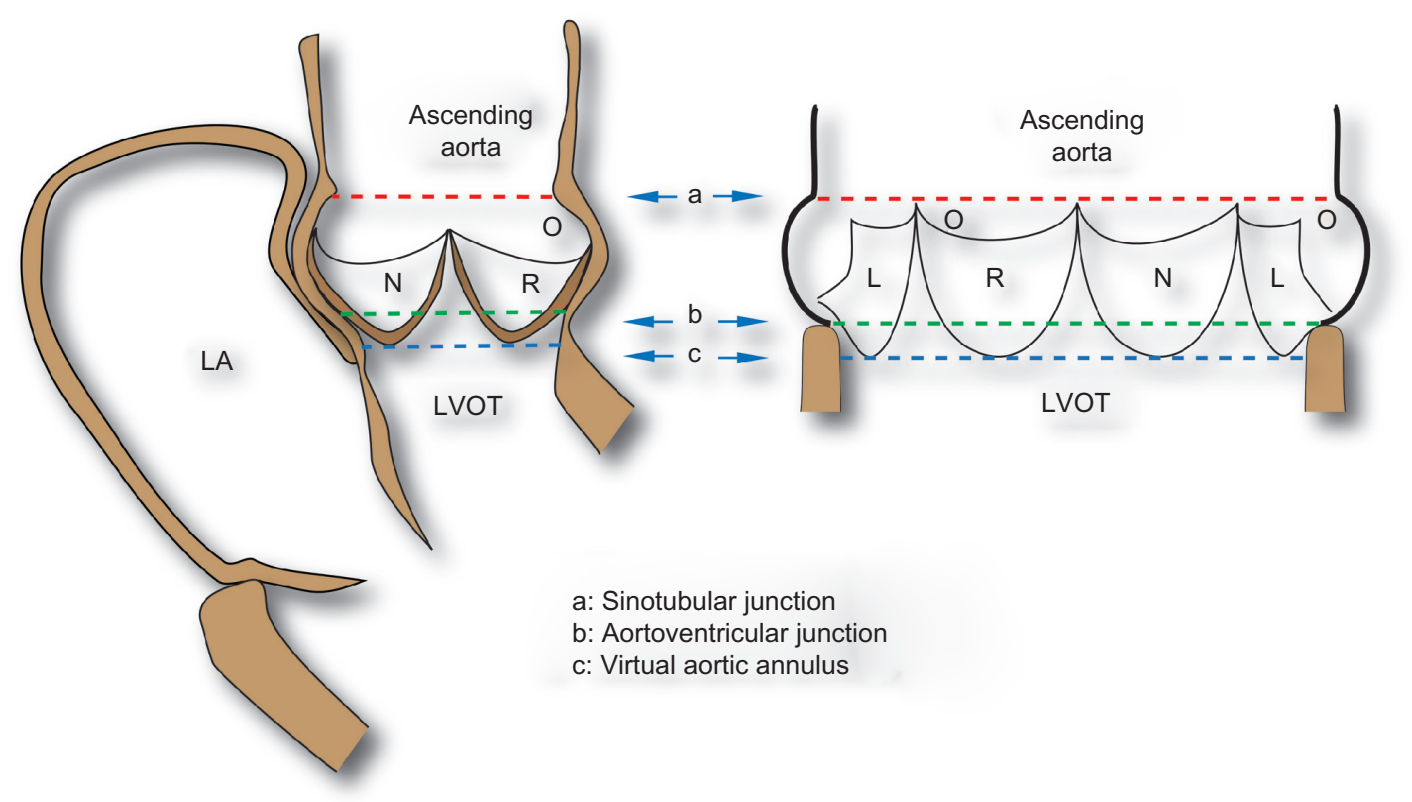

Figure I Diagram illustrating the components of the aortic valve complex. Note the most superior annulus (a) at the sinotubular junction, a virtual annulus at the nadir of the aortic leaflets ([c] this is the dimension used for the TAVR sizing), and a third annulus at the aortoventricular junction (b). The circles in the right and left sinus of Valsalva represent the coronary ostia.

Abbreviations: LA, left atrium; LVOT, left ventricular outflow tract; L, left coronary cusp; R, right coronary cusp; N, noncoronary cusp; TAVR, transcatheter aortic valve replacement. 
that is mobilized when a prosthesis is deployed. Determining the sinus of Valsalva diameter and volume will help predict when an excess amount of calcium may obliterate the sinus of Valsalva and potentially the coronary ostia. In addition, the maximal length of the right and left coronary cusps needs to be known in relation to the distance between the nadirs of the cusps to the coronary ostia. A long leaflet in a shallow sinus of Valsalva has the potential to obliterate the coronary ostia when a transcatheter prosthetic aortic valve is deployed.

\section{Imaging techniques in aortic stenosis}

The decision to intervene in a patient with AS is based on the presence of symptoms that can be attributed to the $\mathrm{AS}$, the demonstration of severe AS, and the characterization of a suitable aortic valve complex for TAVR deployment. The hemodynamic evaluation of AS severity in the cardiac catheterization laboratory has been replaced by hemodynamic data derived from echo and Doppler methods. The American Heart Association/American College of Cardiology and the European Society of Cardiology guidelines define severe AS by echocardiography as a valve area of less than $1 \mathrm{~cm}^{2}$, an aortic valve area index of $0.6 \mathrm{~cm}^{2}$, a mean transvalvular gradient of $40 \mathrm{mmHg}$, and a peak transvalvular velocity of $4 \mathrm{~m} /$ second. $^{3,17}$ Transthoracic echocardiography readily provides this information, as illustrated in Figure 2.

In addition to transthoracic two-dimensional (2-D) echocardiography and spectral Doppler imaging, several imaging tools have emerged for the evaluation and management of patients with calcific AS being considered for or undergoing TAVR, and are the main topic of this review. Table 1 highlights the imaging tools and the first- and secondline tests used in TAVR procedures.
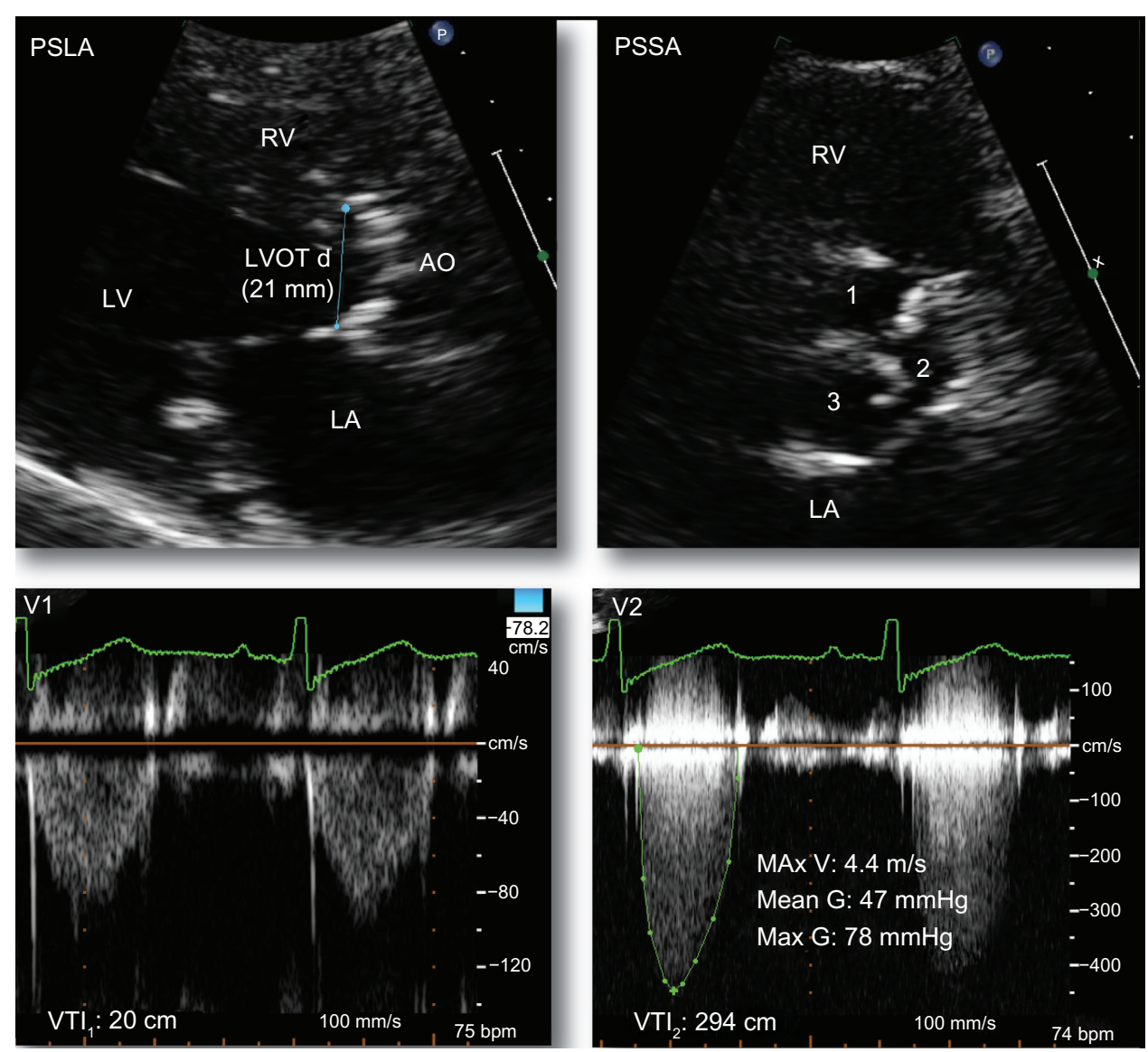

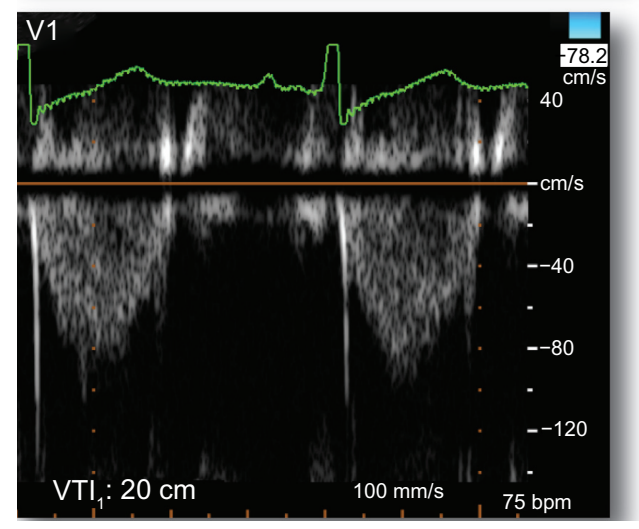

$\mathrm{AVA}=\left[\pi \times(\mathrm{LVOT} \mathrm{d} / 2)^{2} \times \mathrm{VTI}_{1}\right] / \mathrm{VTI}_{2}$

$\mathrm{AVA}=\left[3.14 \times(21 / 2)^{2} \times 20\right] / 294=0.7 \mathrm{~cm}^{2}$

Figure 2 Conventional echo/Doppler measurements in aortic stenosis. Transthoracic parasternal long axis (PSLA) and short axis (PSSA) 2-D echocardiogram in a patient with severe calcific trileaflet aortic stenosis. Severe aortic stenosis is established by determining a maximal transaortic velocity (Max V) of $4.4 \mathrm{~m} /$ second, a mean gradient (Mean G) of $47 \mathrm{mmHg}$, and a maximal gradient (Max G) of $78 \mathrm{mmHg}$. Calculation of the aortic valve area (AVA) by the continuity equation requires: measuring the diameter of the left ventricular outflow tract (LVOT d), the velocity time integral $\left(\mathrm{VTI}_{1}\right)$ in the outflow tract $\left(\mathrm{V}_{1}\right)$ and the velocity time integral $\left(\mathrm{VTI}_{2}\right)$ across the aortic valve $\left(\mathrm{V}_{2}\right)$.

Notes: I, Right coronary cusp; 2, left coronary cusp; 3, noncoronary cusp.

Abbreviations: LV, left ventricle; RV, right ventricle; Ao, aorta; LA, left atrium. 
Table I Imaging techniques in transcatheter aortic valve replacement

\begin{tabular}{|c|c|}
\hline Technique & Highlights: first- and second-line tools \\
\hline $\begin{array}{l}\text { Conventional echo/Doppler } \\
\text { methods }\end{array}$ & $\begin{array}{l}\text { - Two-dimensional morphologic evaluation of the aortic valve and Doppler-derived mean and peak gradients, as well as } \\
\text { continuity-equation effective aortic valve area } \\
\text { - Preferred tool for the preprocedural assessment of aortic stenosis severity } \\
\text { - Standard tool for aortic annular dimensions } \\
\text { - Color Doppler is the current standard to assess postprocedural aortic insufficiency }\end{array}$ \\
\hline RT 3-D echocardiography & $\begin{array}{l}\text { - Functional/morphologic characterization of the aortic valve complex } \\
\text { - Better estimate of aortic valve area and aortic annular dimensions } \\
\text { - Real-time pre-, intra-, and post-TAVR intervention } \\
\text { - Provides improved accuracy in aortic annular dimensions } \\
\text { - Supports fluoroscopy during TAVR deployment }\end{array}$ \\
\hline Computed tomography & $\begin{array}{l}\text { - Volumetric technique that provides multiplanar reorientation of key aortic valve structural components } \\
\text { - Given the ovoid shape of the aortic valve annulus, volumetric three-dimensional CT provides accurate } \\
\text { measurements of both the major and minor axes of the annulus, and the area } \\
\text { - Best tool to determine size and plaque burden of abdominal aorta and iliac and femoral arteries } \\
\text { - Best tool to determine aortic valve calcium burden } \\
\text { - Becoming the standard for aortic annular measurements } \\
\text { - CT plays a fundamental role in predicting the best C-arm orientation for the fluoroscopic angle to be used at time of } \\
\text { prosthesis deployment }\end{array}$ \\
\hline Cardiac MRI & $\begin{array}{l}\text { - CMR is able to quantify the severity of AS, the presence of other types of outflow stenosis, such as HOCM, and } \\
\text { subaortic membranes or CABG graft patency when selecting TAVR patients }\end{array}$ \\
\hline $\begin{array}{l}\text { Fluoroscopy and advanced } \\
\text { cineangiography }\end{array}$ & $\begin{array}{l}\text { - Fluoroscopy is the best tool to navigate catheters and devices during TAVR procedures } \\
\text { - Advanced cineangiography facilitates radiographic images perpendicular to the valve plane in an optimal projection } \\
\text { for TAVR deployment }\end{array}$ \\
\hline
\end{tabular}

Abbreviations: $\mathrm{CT}$, computed tomography; MRI, magnetic resonance imaging; TAVR, transcatheter aortic valve replacement; CABG, coronary artery bypass surgery; AS, aortic stenosis; HOCM, hypertrophic obstructive cardiomyopathy; RT 3-D, real time three dimensional; CMR, cardiac magnetic resonance.

\section{3-D echocardiography in aortic stenosis}

The standard echocardiographic assessment of AS consists of 2-D morphologic evaluation of the aortic valve and Dopplerderived mean and peak gradients, as well as the continuityequation effective aortic valve area. ${ }^{18} 2$-D echocardiography usually distinguishes a bileaflet from a trileaflet aortic valve, and permits fair characterization of calcium burden and leaflet motion restriction; however, the spatial resolution of 2-D echo transesophageal echocardiography (TEE) is usually inadequate to measure the aortic valve orifice area accurately. Even with 2-D TEE, which provides better spatial resolution, it is difficult to select the precise plane that transects the smallest aortic valve orifice.

Real-time (RT) 3-D TEE allows for an en-face "surgeon's view" of the aortic valve, providing detailed morphologic description of the aortic valve ${ }^{19}$ (Figure 3). RT 3-D TEE is emerging as the preferred echocardiographic tool for the morphologic and functional characterization of the stenotic aortic valve ${ }^{20-25}$ (Figure 4). RT 3-D TEE facilitates the obtaining of the cross-sectional plane where the aortic valve area is smallest, usually at the tips of the leaflets. ${ }^{24}$ The 3-D-based biplane (xPlane mode) or multiplane (3-D zoom or fullvolume modes) can be used for this purpose (Figure 5).

In addition to improving the planimetric measure of the aortic valve orifice, RT 3-D TEE can improve the estimation of the aortic valve area from the continuity equation, by providing a better measure of the true LV outflow tract (LVOT) area. It is now well recognized that the LVOT frequently has an oval shape and is not a perfect circle. RT 3-D TEE allows for a better characterization of the oval-shaped LVOT, ${ }^{26}$ thereby improving the accuracy of the continuity equationderived aortic valve area. ${ }^{27-29}$

\section{3-D echocardiography in patients undergoing TAVR procedures}

TAVR demands a multimodality-imaging team-based approach (involving echocardiography, angiography and fluoroscopy, CT angiography [CTA], and cardiac magnetic resonance imaging [CMRI]) throughout preprocedural planning, intraprocedural guidance, immediate evaluation of results, and long-term follow-up. ${ }^{13}$ Patients undergoing TAVR require the expert use of various imaging modalities, each of which has its own strengths and limitations. In this section, we highlight the pivotal role echo/Doppler imaging and RT 3-D TEE play in the continuum of care of patients undergoing TAVR.

\section{Preprocedural planning}

RT 3-D TEE plays a pivotal role in assessing aortic valve annulus dimensions, sinus of Valsalva distance, sinotubular 


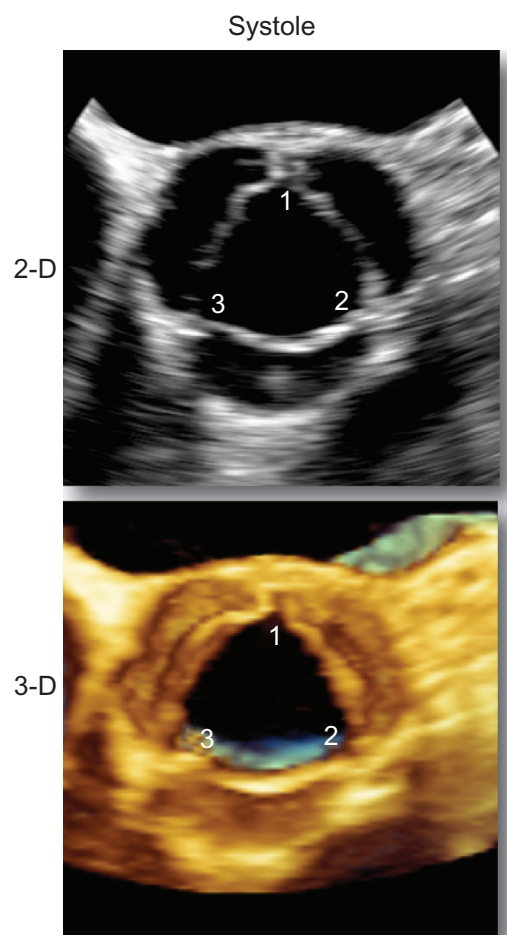

Commissures

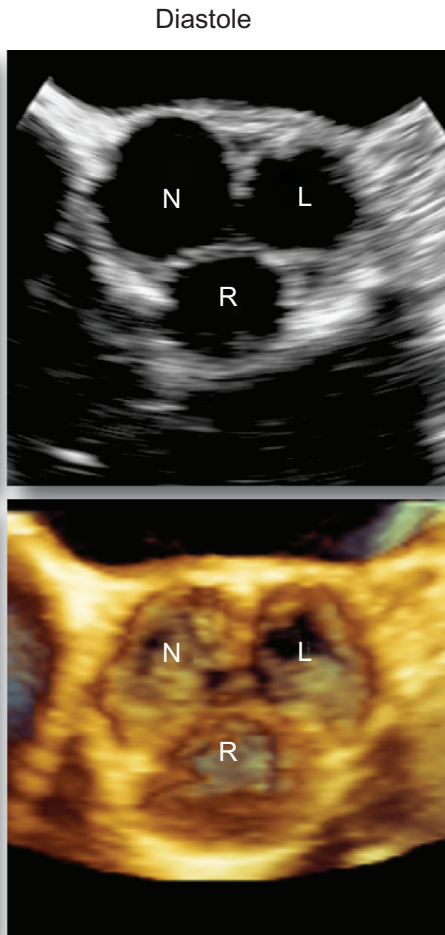

Cusps

Figure 3 Normal aortic valve - short axis. Two-dimensional (2-D) and three-dimensional (3-D) transesophageal echocardiograms of a normal aortic valve, as seen from the aorta (surgeon's view). Note the additional depth information gained from the volumetric 3-D rendition.

Notes: I, Commissure between $\mathrm{L}$ and $\mathrm{N}$ coronary cusps; 2 , commissure between $\mathrm{L}$ and $\mathrm{R}$ coronary cusps; 3 , commissure between $\mathrm{R}$ and $\mathrm{N}$ coronary cusps.

Abbreviations: $\mathrm{N}$, noncoronary cusp; L, left coronary cusp; R, right coronary cusp.

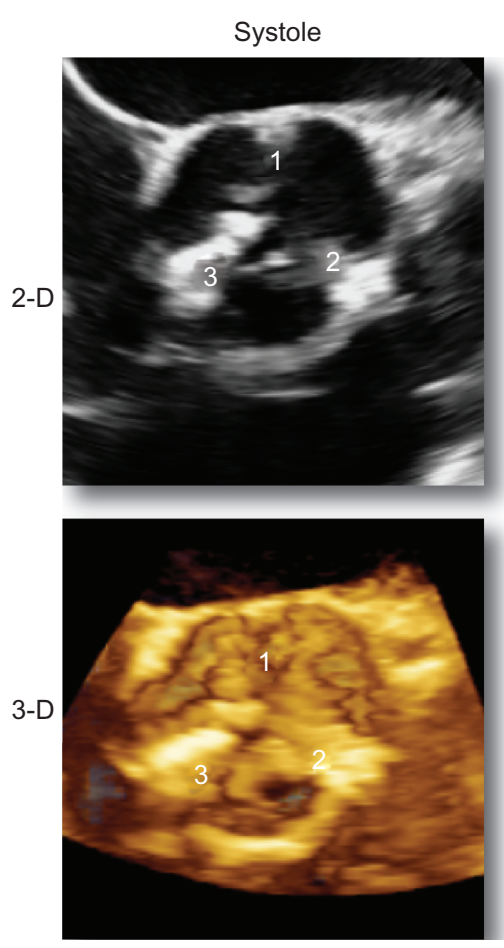

Commissures
Diastole
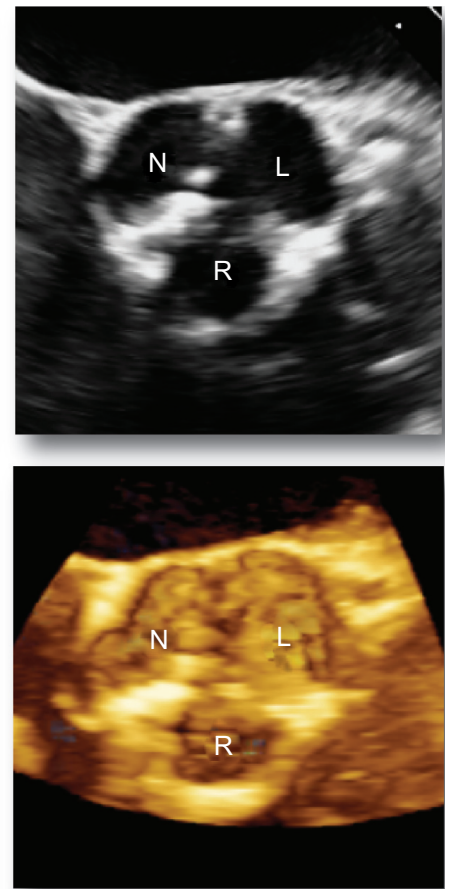

Cusps

Figure 4 Aortic stenosis - short axis. Two dimensional (2-D) and three-dimensional (3-D) transesophageal echocardiograms of the aortic valve, as seen from the aorta (surgeon's view) in a patient with severe calcific aortic stenosis. Note the additional depth information gained from the volumetric 3-D rendition.

Notes: I, Commissure between $\mathrm{L}$ and $\mathrm{N}$ coronary cusps; 2 , commissure between $\mathrm{L}$ and $\mathrm{R}$ coronary cusps; 3 , commissure between $\mathrm{R}$ and $\mathrm{N}$ coronary cusps. Abbreviations: $\mathrm{N}$, noncoronary cusp; L, left coronary cusp; R, right coronary cusp. 


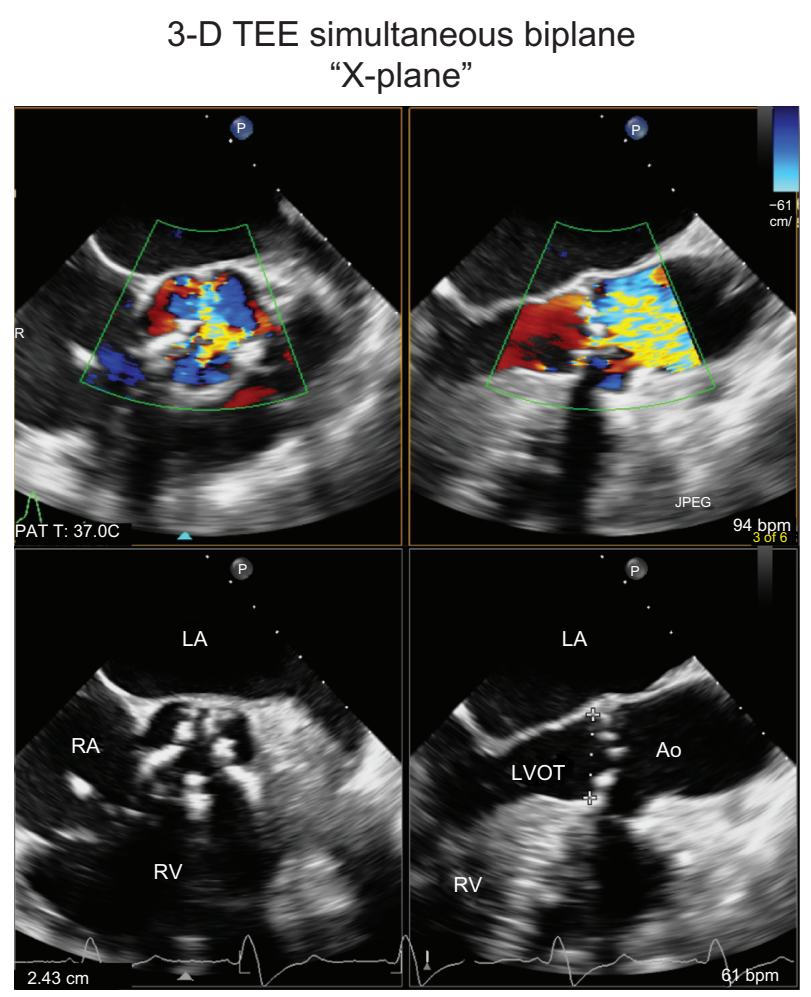

Figure 5 Aortic stenosis - long axis. Three-dimensional (3-D)-derived simultaneous Biplane ("X-Plane") transesophageal views of a normal aortic valve complex seen in the longitudinal plane during systole. The upper panels illustrate with color Doppler the turbulent flow through the narrow aortic valve orifice. The lower panels illustrate how orthogonal views can be used to measure the aortic annulus (dotted line). Abbreviations: LVOT, left ventricular outflow tract; RV, right ventricle; RA, right atrium; Ao, aorta; LA, left atrium.

junction dimension, and left main trunk ostia height ${ }^{30,31}$ (Table 2 and Figure 6). In addition, RT 3-D TEE helps immensely in characterizing the LVOT size and shape and demonstrating the absence of subvalvular obstruction. ${ }^{26}$ All of these parameters are used to determine the feasibility of a TAVR procedure and the appropriate prosthetic size. ${ }^{32,33}$

Important findings regarding calcium deposits on or around the aortic valve include extent and asymmetry of leaflet involvement, mobile calcium deposits potentially more likely to embolize, and extension of calcium deposits in the LVOT and sinotubular junction. RT 3-D TEE facilitates this characterization.

\section{Intraprocedural guidance}

TAVR is heavily dependent on fluoroscopic and angiographic methods. ${ }^{34}$ RT 3-D TEE supplements these X-ray-based procedures by providing a 3-D rendition of the aortic valve complex, facilitating maneuvering of the delivery system and proper positioning of the aortic prosthesis ${ }^{32}$ (Table 2 and Figure 7). RT 3-D TEE also plays a central role in the prompt recognition
Table 2 Role of echo/Doppler and real-time three-dimensional transesophageal echocardiography in TAVR

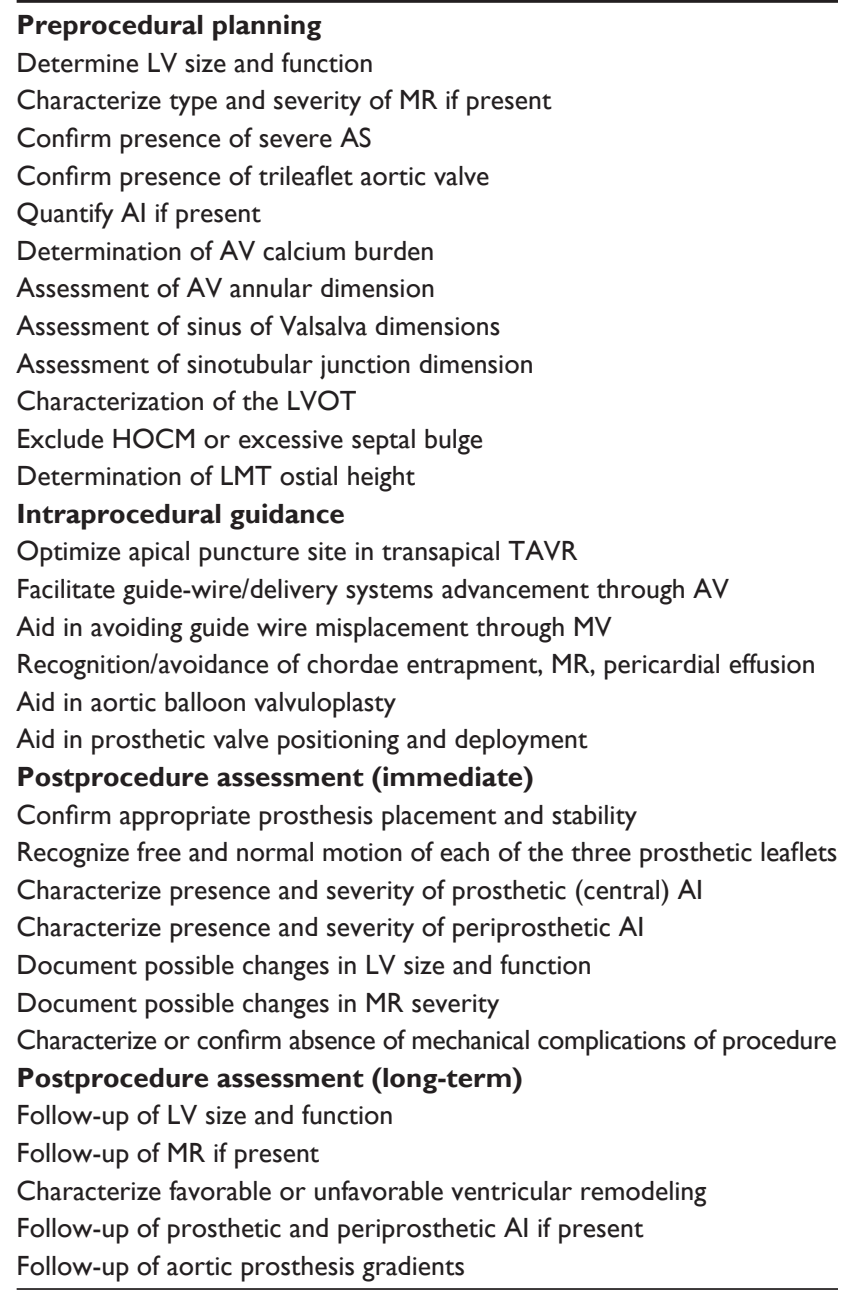

Abbreviations: LVOT, left ventricular outflow tract; TAVR, transcatheter aortic valve replacement; $\mathrm{AV}$, aortic valve; $\mathrm{MR}$, mitral regurgitation; $\mathrm{Al}$, aortic insufficiency; $\mathrm{LV}$, left ventricle; LMT, left main trunk; HOCM, hypertrophic obstructive cardiomyopathy; AS, aortic stenosis; MV, mitral valve.

of intraprocedural TAVR complications and facilitates the appropriate management of these potential problems.

\section{Postprocedure assessment}

Echocardiographic methods are uniquely suited for the immediate and long-term postprocedure assessment of TAVR procedures. Echocardiography and Doppler methods permit a detailed functional and structural assessment of the newly deployed transcatheter aortic bioprosthesis (Table 2 and Figure 7). Immediately after the prosthesis has been deployed, 2-D and 3-D TEE permit confirmation of appropriate prosthesis position and stability, evaluation of normal leaflet motion, and characterization of the presence, severity, and location of prosthetic and periprosthetic aortic regurgitation (Figure 8). Long-term post-TAVR assessment also relies 


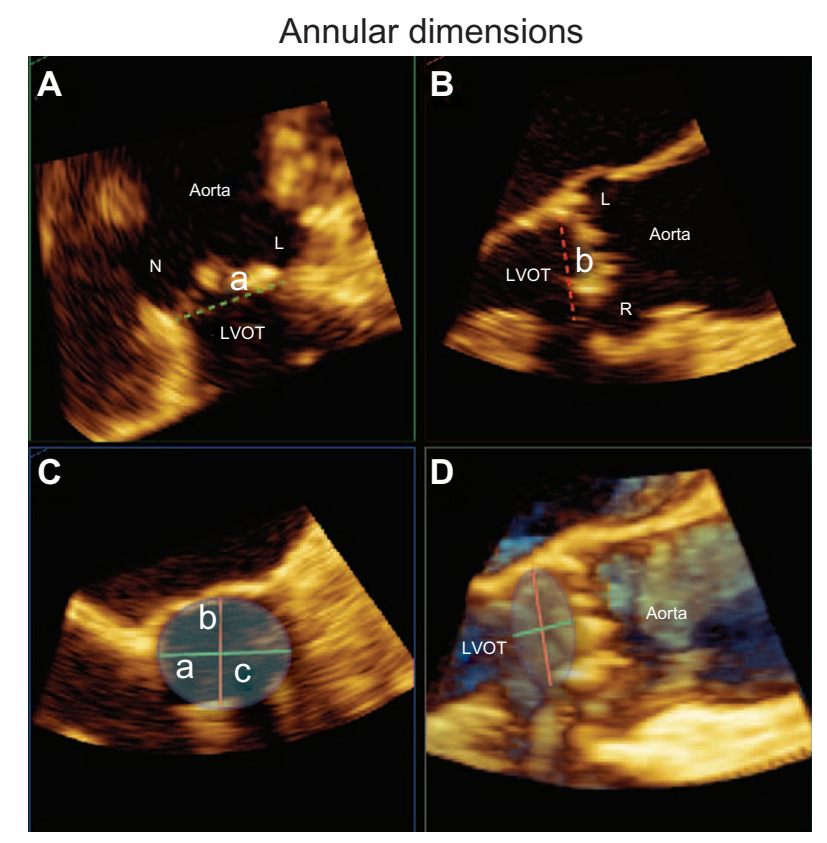

Figure 6 (A-D) Three-dimensional (3-D) TEE annular dimensions. Multiplane reconstruction from a real-time 3-D TEE data set of the aortic valve complex. (D) 3-D volumetric rendition of the aortic valve complex in systole. From this, three orthogonal planes are selected to obtain the true aortic valve annulus dimensions and area. (A) The coronal plane, from which the medial-lateral annular dimension is obtained (a, dotted green line). (B) The sagittal plane, from which the anteroposterior annular dimension is obtained (b, dotted red line). (C) The transverse plane, from which the circumference or area of the aortic annulus is obtained (c, transparent ellipse)

Abbreviations: TEE, transesophageal echocardiography; LVOT, left ventricular outflow tract; $\mathrm{N}$, noncoronary sinus of Valsalva; L, left coronary sinus of Valsalva; $\mathrm{R}$, right coronary sinus of Valsalva. mainly on echocardiographic methods. LV size and function and presence and severity of mitral regurgitation are assessed; prosthesis stability, leaflet motion, prosthetic gradients, and presence and degree of prosthetic and periprosthetic aortic regurgitation are evaluated by echocardiography.

\section{Computed tomographic angiography}

A multimodality imaging approach to aortic valve stenosis incorporates CTA as a volumetric technique that provides multiplanar reorientation of key aortic valve structural components. ${ }^{35}$ Such features of CTA allow careful examination of valve characteristics (bicuspid valves or stenotic trileaflet valves), sinuses, and aorta under scrutiny for either surgical or percutaneous valve replacement. This is in addition to assessment of iliofemoral access for percutaneous procedures and the position of the cardiac apex for a transapical approach. ${ }^{36,37}$

Initial evaluation of potential candidates usually employs 2-D TEE to assess the severity of stenosis and actual valve characteristics. ${ }^{38,39}$ However, the degree of calcific degeneration of the stenotic valve limits the exact determination of a bicuspid versus trileaflet valve in many cases. Definition of structural tissue compared to calcium is a strength of CTbased technologies. The extent and severity of aortic valve leaflet calcification can be quantified by either contrast or

\section{Severe AS-Pre-TAVR}
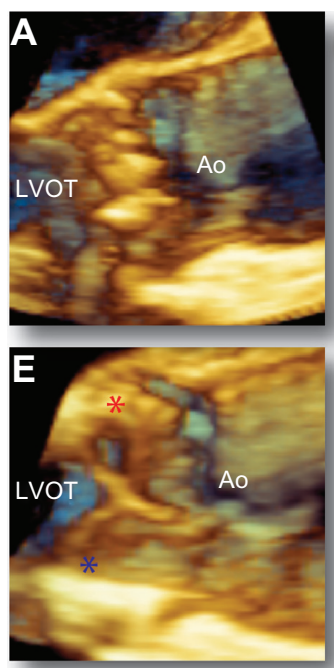

Post TAVR implantation
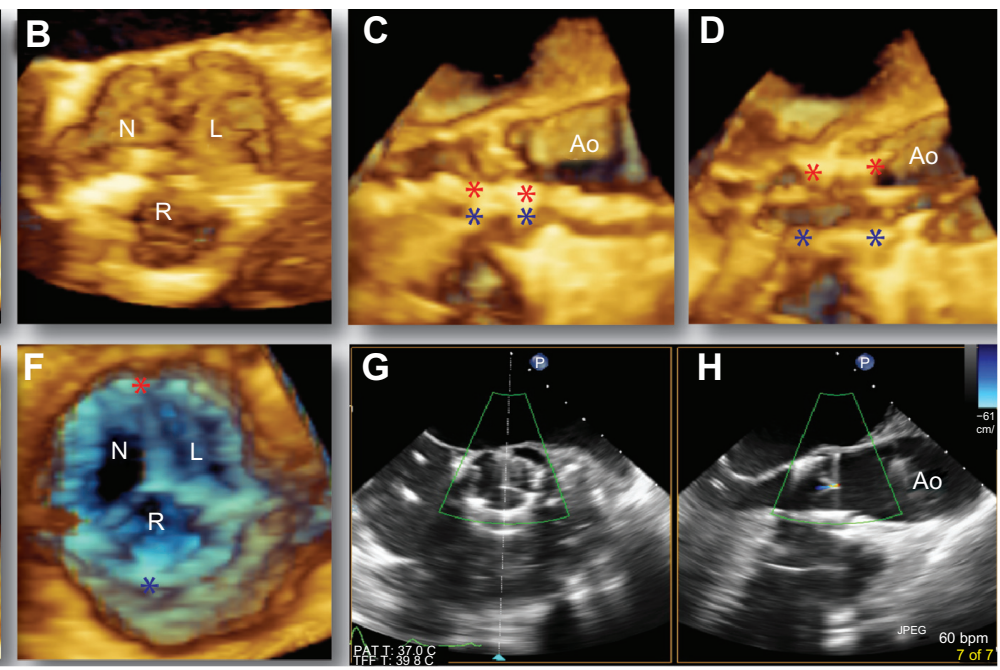

Post TAVR-No Al

Figure 7 (A-H) Real-time three-dimensional (3-D) TEE guidance of TAVR. (A and B) Long- and short-axis views of a stenotic aortic valve prior to TAVR. (C and D) Intra-procedure TAVR guidance: in (C) the crimped prosthesis is positioned in the middle of the stenotic aortic orifice; in (D) the prosthesis has been balloon-expanded to its final size and position. (E and $\mathbf{F}$ ) Long- and short-axis depictions of the deployed prosthesis during diastole. (G, $\mathbf{H})$ 3-D-derived simultaneous biplane images of the deployed prosthesis, with color Doppler demonstrating absence of prosthetic or periprosthetic aortic regurgitation.

Abbreviations: TEE, transesophageal echocardiography; TAVR, transcatheter aortic valve replacement; AS, aortic stenosis; Al, aortic insufficiency; N, noncoronary cusp; L, left coronary cusp; R, right coronary cusp; LVOT, left ventricular outflow tract; Ao, aorta. 


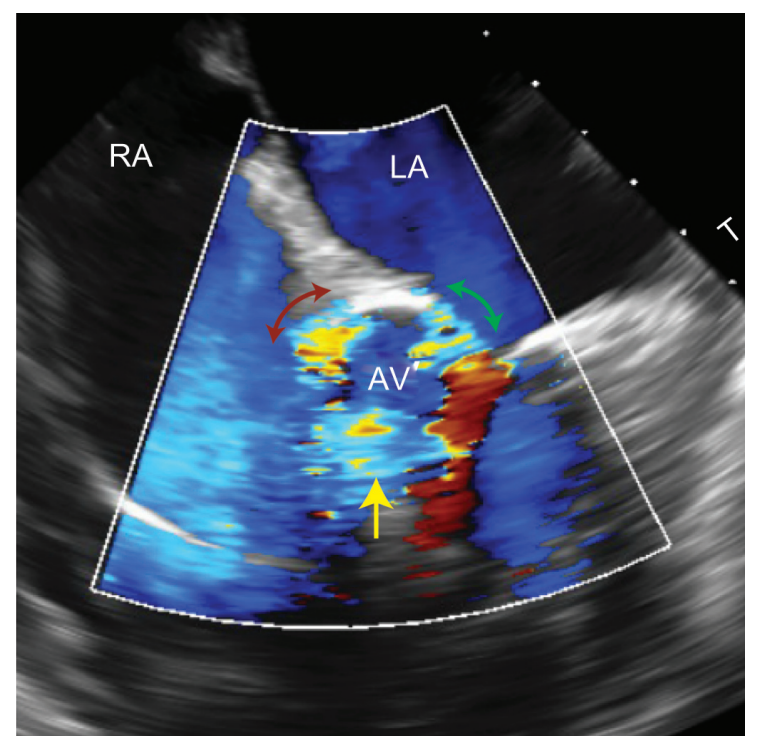

Figure 8 Post-TAVR periprosthetic insufficiency. Color Doppler mid-esophageal TEE at the level of the aortic valve obtained immediately post-TAVR deployment and illustrating the presence of central (prosthetic) aortic regurgitation (yellow arrow) and two areas of periprosthetic aortic regurgitation (red and green arrows). The degree of periprosthetic aortic insufficiency was felt to be moderate by the percentage (about 25\%) of the annular circumference having a periprosthetic leak. A second balloon inflation decreased the degree of leak to only mild.

Abbreviations: TAVR, transcatheter aortic valve replacement; TEE, transesophageal echocardiography; RA, right atrium; LA, left atrium; AV, aortic valve.

noncontrast images, although leaflet morphology requires contrasted studies. Once oriented in orthogonal planes to view the commissures, it is easy to define two or three cusps. Functional bicuspid valves are seen frequently and maintain a triangular shape to the valve opening, but the fused raphe results in a nonuniform triangle. Since the definition of a bicuspid valve is considered a limitation of percutaneous treatment of aortic valve stenosis, then correct identification is important. An unequal but still triangular orifice seen with functional bicuspid valve may still allow a percutaneous TVAR approach. In conjunction with the orifice shape is the actual surface area at the end of systole, defining the severity of stenosis. Generally, the orifice and leaflet tips are visualized well, despite higher heart rates in patients with severe AS; however, heart rates over $100 \mathrm{bpm}$ may be problematic for CTA. Planimetry of the orifice is well correlated between anatomic assessments of AS by CT compared to functional assessments by echocardiography. ${ }^{38}$

Critical issues that must be evaluated before a decision for TAVR or surgical AVR relate to preoperative risk assessment, including such findings as a "porcelain aorta" or large annular size. Commercial-release TAVR is limited to nonoperative or very high-surgical-risk individuals, in which the valve device sizes are limited to 23,26 , or $29 \mathrm{~mm} .{ }^{4,5}$ Therefore, CTA provides a technique to allow detailed interrogation of the aortic valve annulus and the relationship to the coronary arteries, which helps to either include or exclude an individual for a TAVR procedure. ${ }^{40}$

Given the ovoid shape of the aortic valve annulus, volumetric 3-D CT provides accurate measurements of both the major and minor axes of the annulus and the area (Figure 9). These measurements are critical for choosing an appropriately sized device. An oversized prosthesis can result in annular and/or sinotubular junction rupture or coronary artery occlusion, ${ }^{41,42}$ whereas under sizing the valve device may result in significant perivalvular leaks and aortic insufficiency, or more catastrophically device migration and embolization. ${ }^{36,40,42}$ Definition of the correct annular orientation and axis is complex. Shown in Figure 9 are the similarities and differences between 3-D TEE and CTA using a similar multiplanar reformation technique. ${ }^{15,30}$ The CTA images are less affected by calcium than are the echocardiographic images, leading to better determination of the annular size. Early device trials solely used echocardiography to determine annular size, but more recently CTA has become the more accepted standard for annular measurements when technically adequate..$^{30,40}$ Positioning of the two axes requires careful adjustment of the angulation of this 3-D structure at the systole, due to the annular insertion, as described previously.

Once the angles are set, panning through the aortic valve short-axis image set is performed until the plane just below the insertion of the three-cusp position is noted. At this point, the area is determined, from which the major and minor axes are measured. Some investigators have used the mean annular measurement for deciding appropriate device size, while others use the area of the annulus. A rough guide is that the upper-limit area of $420 \mathrm{~mm}^{2}$ is used for $23 \mathrm{~mm}$ valves, and $530 \mathrm{~mm}^{2}$ is used for the $26 \mathrm{~mm}$ valve..$^{30,40,41}$ Lastly, the clear identification of the coronary ostia allows determination of the distance between the annulus and the ostium as it relates to the length of each leaflet. Concern about coronary ostium occlusion is generated when either the leaflet length is longer than the height (annulus to ostium) or the height is less than $10 \mathrm{~mm}$. Orthogonal image reorientation using CTA provides accurate measurement of these key parameters. ${ }^{43}$

Routinely, CT can assess the thoracic aorta for aneurysmal dilatation, stenosis to the major vessel, and large ascending or arch atheromatous plaques at risk of embolization during the procedure ${ }^{35}$ (Figure 10). The relationship between the aortic root and LV cavity is also carefully scrutinized for potential excessive angulation or mitral valve chordal entrapment when a transapical approach is entertained. 

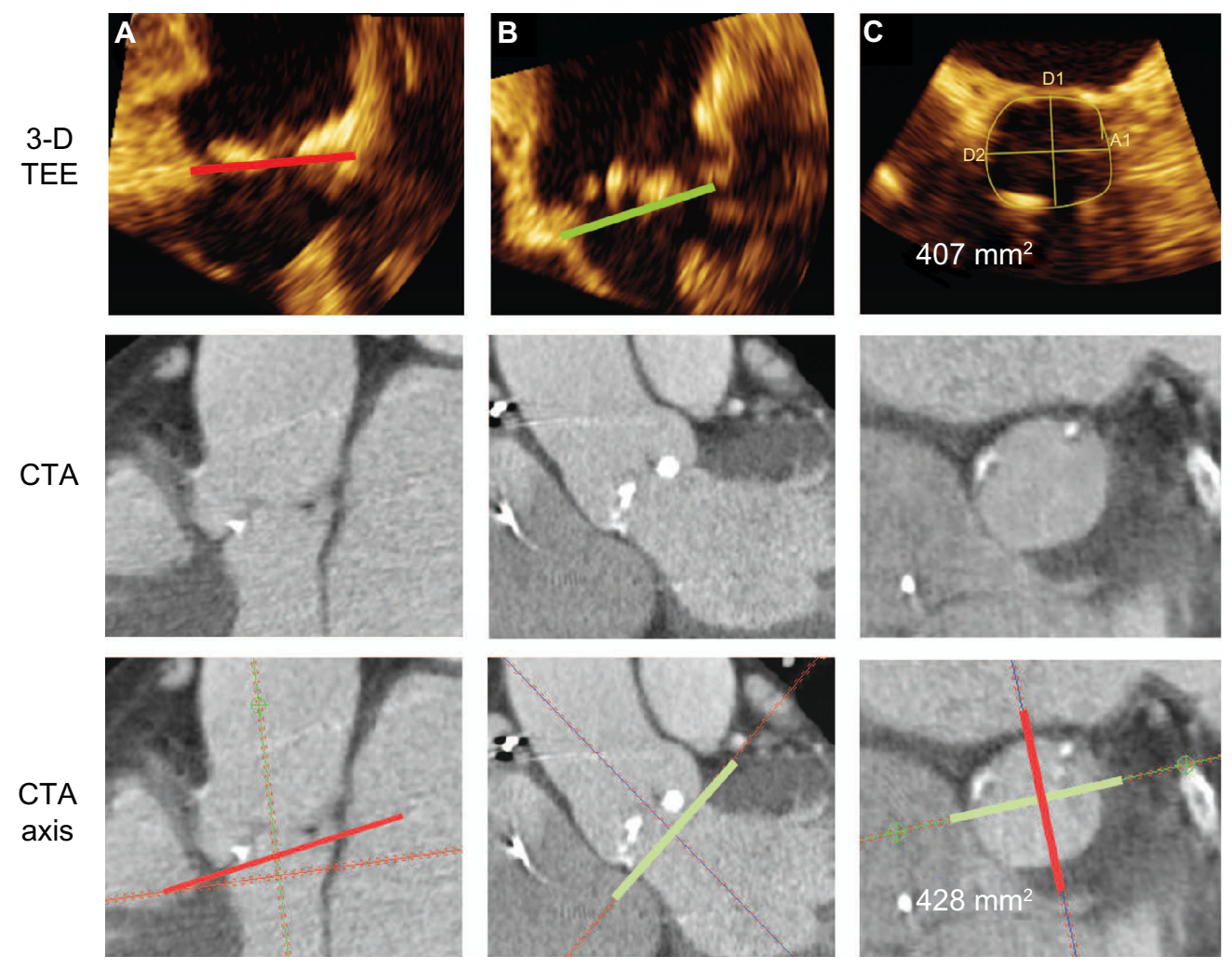

Figure 9 (A-C) Comparison between three-dimensional (3-D) TEE (top) and CTA (middle and bottom) assessment of orthogonal planes is shown in anterior/posterior plane $(\mathbf{A})$, medial/lateral long axis $(\mathbf{B})$, and aortic valve in short axis $(\mathbf{C})$. The bottom image shows the adjustment for the correct aortic valve annular plane. Note in $(\mathbf{C})$ the annular area by each technique is similar, but the major and minor axes are more difficult to identify by 3-D TEE due to calcium degradation of the image with ultrasound. Abbreviations: TEE, transesophageal echocardiography; CTA, computed tomographic angiography.

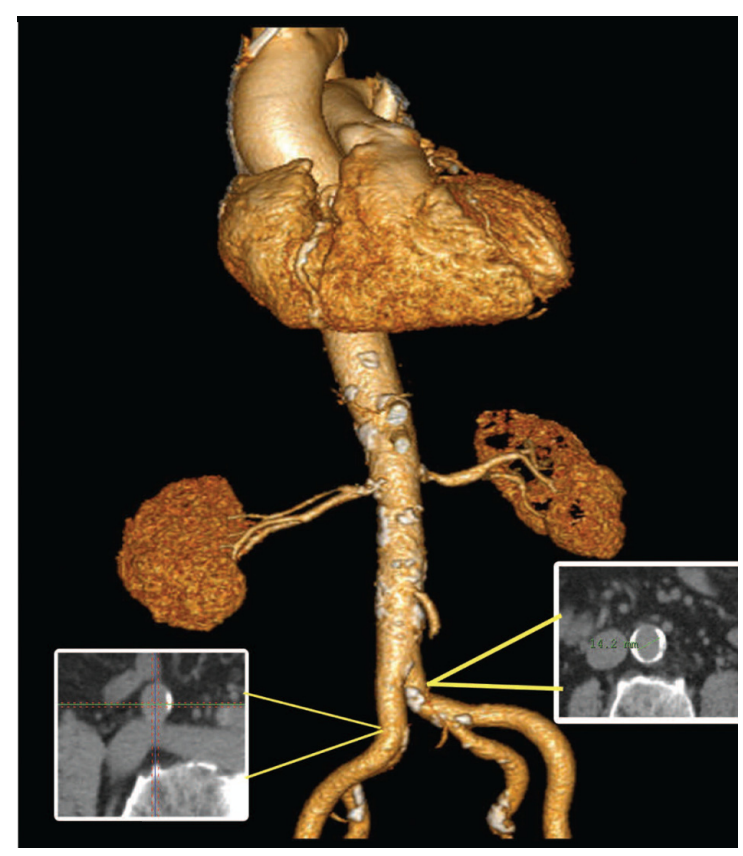

Figure 10 Shown are volumetric three-dimensional reconstructions of the entire vascular tree in a patient screened for TAVR. Note in the small focal images the greater than $50 \%$ circumferential calcium (left) compared to the normal vessel (right). This finding could limit peripheral arterial access for a TAVR procedure, since vascular stretch is impaired.

Abbreviations: TAVR, transcatheter aortic valve replacement.
Distal to the thoracic aorta, the abdominal aorta and iliac vessels extending to the common femoral artery are assessed for size and plaque burden (Figure 10). The large-diameter delivery sheaths required for device deployment (between 18 and 28 French, minimum of $7-9 \mathrm{~mm}$ for 23 and $26 \mathrm{~mm}$ valves, respectively) necessitate accurate femoral-to-iliac artery and distal aortic measurements. These vessels must "stretch" around these large sheaths for delivery of the valve through the aorta, across the arch, and ultimately across the native valve. CT provides information about the circumferential nature of calcification, plaque stenosis, and minimal luminal diameter. ${ }^{35,36}$ Circumferential calcification does not stretch, thereby increasing the risk of arterial dissection or perforation. The entire course of the vascular tree can be assessed using volumetric 3-D-rendered displays of vessel angulation and tortuosity. Different methods have been proposed to help define the characteristics favorable for peripheral vascular access ${ }^{44}$ but we find that direct orthogonal cross-sectional size measurement is the most useful.

In addition, CT plays a fundamental role in predicting the best $\mathrm{C}$-arm orientation for the fluoroscopic angle to be used at the time of prosthesis deployment (Figure 11). 

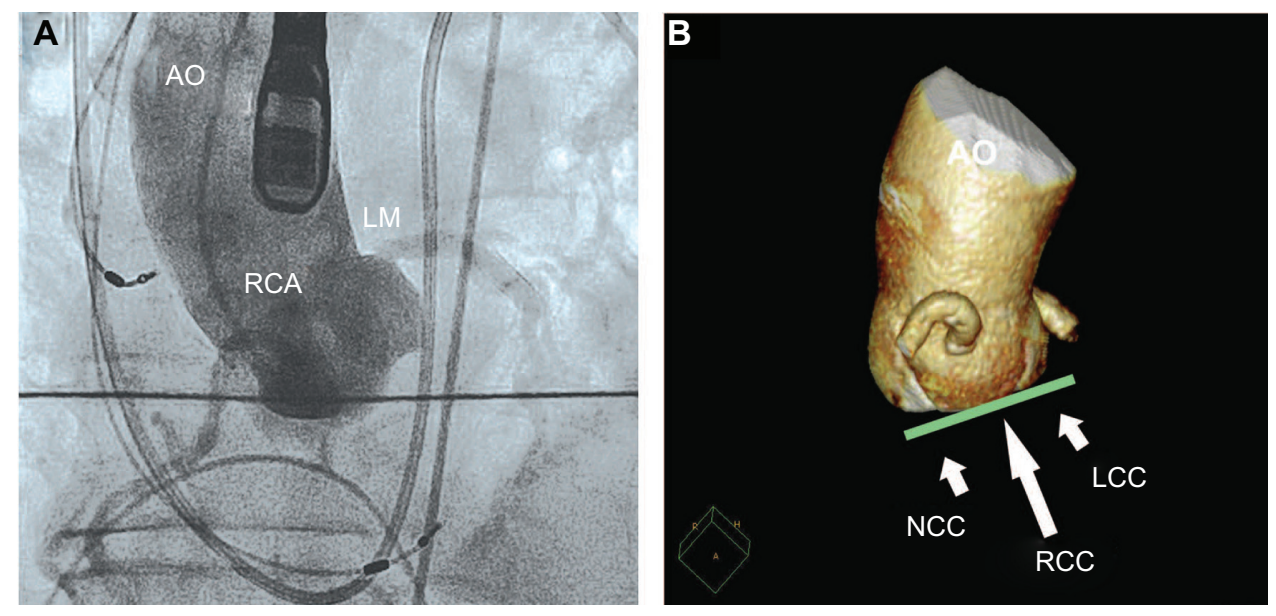

Figure II (A and B) Computed tomography (CT) angiography images can determine the best planar view of the aortic valve cusps. Alignment of the bottoms of the cusps provides a plane that is determined by the cranial/caudal and RAO/LAO (right anterior oblique/left anterior oblique) angulation. The image on the left shows the aortic root (AO) in the best implantation projection, and the corresponding contrast angiogram verifying the position is shown on the right. The individual cusps are labeled on the CT image (right), with the noncoronary cusp (NCC), right coronary cusp (RCC, large arrow), and left coronary cusp (LCC). The coronary ostia labeled in the angiographic image (right coronary artery $[R C A]$ and left main coronary artery [LM]) are similar to the CT three-dimensional image.

This requires careful alignment of the aortic annulus defined by the nadir of each coronary cusp. Clear separation of each cusp allows appropriate alignment of the valve under balloon deployment. Planning appears to reduce total contrast requirements, and with less cinematic angiography the radiation dose is also reduced. ${ }^{36}$

\section{Cardiac magnetic resonance imaging}

CMRI in pure AS is limited, though it still provides useful information in the preprocedural evaluation of TAVR patients by excluding other types of cardiomyopathies, predominant aortic insufficiency, or lesser degree of stenosis. The calcific degenerative process of valvular AS limits CMRI due to signal absorption or susceptibility artifacts causing blackout regions around calcium. Several studies note similar sensitivity for detection of valve stenosis, with close correlation between CMRI, cardiac CT, and TEE measurements of planimetric valve area. CMRI is still able to quantify the severity of AS, the presence of other types of outflow stenosis such as HOCM (hypertrophic obstructive cardiomyopathy) and subaortic membranes or CABG (coronary artery bypass graft) graft patency when selecting TAVR patients. Accurate severity assessment of AS and the valve characteristics (bi- or tricuspid) are also within the scope of a CMRI examination. The location of outflow obstruction and the measurement of peak aortic valve velocity are performed using a phase-contrast imaging sequence, as shown in Figure $12 \cdot{ }^{37,45}$ Cine (steady-state free precession) CMRI can be used to identify the presence of congenital valvular abnormalities, such as bicuspid aortic valves. Also, CMRI quantitates the severity of aortic valve area reduction by planimetry, similar to
CTA. ${ }^{46}$ This correlates with TEE. ${ }^{47} \mathrm{Also}$, AS is often concurrent with valvular regurgitation, and quantitation by CMRI provides an estimate of the volumetric severity of insufficiency by using the phase-contrast technique. ${ }^{48}$

\section{Advanced angiography}

During TAVR, valve positioning and implantation is primarily guided by fluoroscopy. In order to position the valve optimally, it is important to obtain fluoroscopic images that are perpendicular to the valve plane in what is referred to as a "coplanar" view or optimal projection (OP). Ideally, this is a projection that is perpendicular to the valve plane, with all three sinuses of Valsalva clearly visualized. This is often performed through acquisition of multiple aortic root angiograms in various projections to determine the best view, with a significant contrast burden. Recent work has demonstrated that an OP can be determined using volumetric data sets derived from preprocedural multislice CT (MSCT) with good accuracy. ${ }^{36,49}$ In patients with significant renal impairment, the $80-120 \mathrm{~mL}$ of contrast needed for MSCT precludes use of this preprocedurally in those with adequate TEE imaging. As such, alternative strategies for in-room determination of the OP utilize aortic root angiography. These approaches address the impact of differences of patient positioning on the CT scanner compared to the hybrid lab table, especially in the case of patient positioning necessary for transapical TAVR (patient rotated rightward and caudal).

One approach utilizes two aortic root angiograms obtained in orthogonal projections (C-THV; Paieon, New York, NY, USA) using a software solution that identifies a series of 

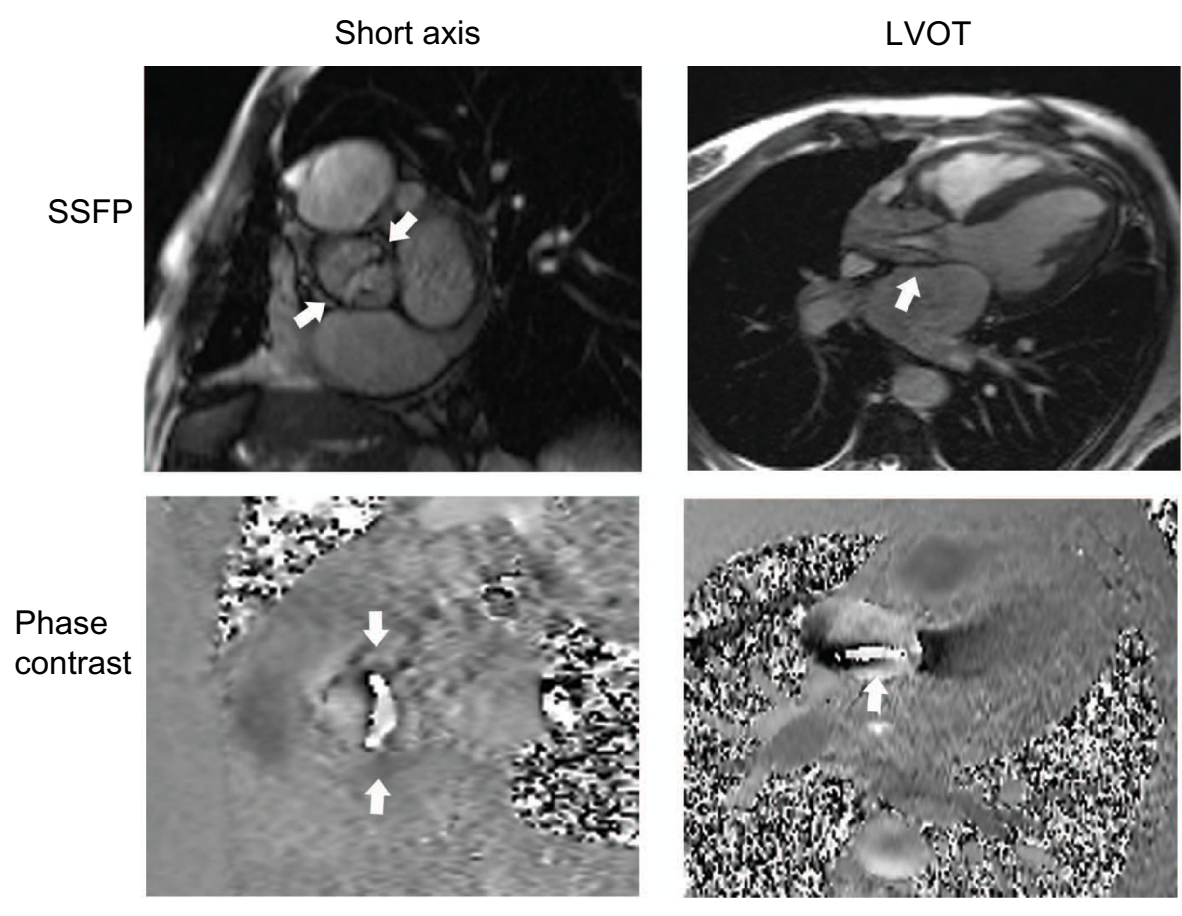

Figure 12 Cardiac magnetic resonance images are shown with cine (SSFP) images on top and phase-contrast (velocity/flow) images shown below. Note the high-velocity flow in the upper-right image, which corresponds to a bicuspid aortic valve (arrows) in the upper left. The aliased velocity of stenosis is shown in the phase-contrast images on the bottom (arrow).

Abbreviations: SSFP, steady-state free precession; LVOT, left ventricular outflow tract.

suitable angles for selection of an OP by the operator. This technology has been evaluated and confirmed to be accurate for OP determination compared to preprocedural MSCT for TAVR, and can be used for positioning as well as at the time of valve implantation. ${ }^{50}$

Another approach is through the acquisition of 3-D volumetric data using in-room $\mathrm{C}$-arm $\mathrm{CT}$ with rotational angiography (RA). This has been well investigated for guidance of peripheral and cerebral interventions. Aortic root angiography is performed using commercial hardware and software during breath hold with rapid ventricular pacing with low-contrast injection rates of 4-8 $\mathrm{mL} / \mathrm{second}$ for a total of 4-6 seconds during rotational angiography. These acquisitions are rapidly converted to 3-D reconstructions that are used to determine the OP for valve implantation. This approach has recently been validated, and it appears that 3-D RA compares favorably to multidetector CT (MDCT) in the determination of OP and final valve-visualization angle..$^{51,52}$ In one recent study, the OP was found to be a mean of $4.9^{\circ}$ LAO (left anterior oblique) and $8.8^{\circ}$ caudal projection for valve implantation. ${ }^{52}$ There was very high correlation of the two approaches, and the mean difference in OP between 3-D RA and MDCT was found to be $4.6^{\circ} \pm 3.5^{\circ}$. Of note, the OP was found to be $3^{\circ}$ more caudal using 3-D RA compared to MSCT, likely related to patient-position differences. Research into the clinical impact of using in-room aortography for procedural planning and outcomes (procedural time, appropriate valve positioning, and perivalvular leak) in TAVR is under investigation at this time.

\section{Conclusion}

Cardiac imaging plays a pivotal role in the contemporary management of patients with calcific AS. Recent advances in 3-D echocardiography allow for precise functional/ morphologic characterization of the aortic valve complex, a better estimate of aortic valve area, and RT pre-, intra-, and post-TAVR intervention imaging guidance. $\mathrm{CT}$ is playing an increasing role in the patient selection and prosthesis-size determination in patients undergoing TAVR procedures. This volumetric technique provides multiplanar reorientation of key aortic valve structural components, providing accurate measurements of both the major and minor axes of the annulus and the area. CMRI is able to quantify the severity of AS, the presence of other types of outflow stenosis, such as HOCM, and subaortic membranes or CABG graft patency when selecting TAVR patients. Advanced angiographic methods, including aortic root angiograms obtained in orthogonal projections and acquisition of 3-D volumetric data using in-room $\mathrm{C}$-arm $\mathrm{CT}$ with rotational angiography, have been developed to optimize appropriate 
valve positioning during TAVR procedures, and presently are undergoing clinical evaluation.

\section{Disclosure}

Dr Salcedo is a consultant for Philips Healthcare. The other authors report no conflicts of interest in this work.

\section{References}

1. Otto CM. Calcific aortic stenosis - time to look more closely at the valve. N Engl J Medicine. 2008;359:1395-1398.

2. Bonow RO, Carabello BA, Chatterjee K, et al. 2008 Focused update incorporated into the ACC/AHA 2006 guidelines for the management of patients with valvular heart disease: a report of the American College of Cardiology/American Heart Association Task Force on Practice Guidelines (Writing Committee to revise the 1998 guidelines for the management of patients with valvular heart disease). Endorsed by the Society of Cardiovascular Anesthesiologists, Society for Cardiovascular Angiography and Interventions, and Society of Thoracic Surgeons. J Am Coll Cardiol. 2008;52:e1-e142.

3. Vahanian A, Alfieri O, Andreotti F, et al. Guidelines on the management of valvular heart disease (version 2012): the Joint Task Force on the Management of Valvular Heart Disease of the European Society of Cardiology (ESC) and the European Association for Cardio-Thoracic Surgery (EACTS). Eur J Cardiothorac Surg. 2012;42:S1-S44.

4. Leon MB, Smith CR, Mack M, et al. Transcatheter aortic-valve implantation for aortic stenosis in patients who cannot undergo surgery. $N E n g l$ J Med. 2010;363:1597-1607.

5. Smith CR, Leon MB, Mack MJ, et al. Transcatheter versus surgical aortic-valve replacement in high-risk patients. N Engl J Med. 2011;364: 2187-2198.

6. Osnabrugge RL, Mylotte D, Head SJ, et al. Aortic stenosis in the elderly: disease prevalence and number of candidates for transcatheter aortic valve replacement: a meta-analysis and modeling study. $J \mathrm{Am}$ Coll Cardiol. 2013;62:1002-1012.

7. Généreux P, Head SJ, Wood DA, et al. Transcatheter aortic valve implantation 10-year anniversary: review of current evidence and clinical implications. Eur Heart J. 2012;33:2388-2398.

8. Tamburino C, Barbanti M, Capodanno D, et al. Comparison of complications and outcomes to one year of transcatheter aortic valve implantation versus surgical aortic valve replacement in patients with severe aortic stenosis. Am J Cardiol. 2012;109:1487-1493.

9. Hayashida K, Bouvier E, Lefevre T, et al. Transcatheter aortic valve implantation for patients with severe bicuspid aortic valve stenosis. Circ Cardiovasc Interv. 2013;6:284-291.

10. Bohula May EA, Faxon D. Transcatheter aortic valve replacement: History and current status. Trends Cardiovasc Med. 2013;23:172-178.

11. Reynolds MR, Magnuson EA, Lei Y, et al. Health-related quality of life after transcatheter aortic valve replacement in inoperable patients with severe aortic stenosis. Circulation. 2011;124:1964-1972.

12. Pibarot P, Dumesnil JG. Low-flow, low-gradient aortic stenosis with normal and depressed left ventricular ejection fraction. J Am Coll Cardiol. 2012;60:1845-1853.

13. Bloomfield GS, Gillam LD, Hahn RT, et al. A practical guide to multimodality imaging of transcatheter aortic valve replacement. JACC Cardiovasc Imaging. 2012;5:441-455.

14. Muraru D, Badano LP, Vannan M, Iliceto S. Assessment of aortic valve complex by three-dimensional echocardiography: a framework for its effective application in clinical practice. Eur Heart J Cardiovasc Imag. 2012;13:541-555.

15. Calleja A, Thavendiranathan P, Ionasec RI, et al. Automated quantitative 3-dimensional modeling of the aortic valve and root by 3-dimensional transesophageal echocardiography in normals, aortic regurgitation, and aortic stenosis: comparison to computed tomography in normals and clinical implications. Circ Cardiovasc Imaging. 2013;6:99-108.
16. Piazza N, de Jaegere P, Schultz C, Becker AE, Serruys PW, Anderson RH. Anatomy of the aortic valvar complex and its implications for transcatheter implantation of the aortic valve. Circ Cardiovasc Interv. 2008;1:74-81.

17. American College of Cardiology/American Heart Association Task Force on Practice G, Society of Cardiovascular A, Society for Cardiovascular A, et al. ACC/AHA 2006 guidelines for the management of patients with valvular heart disease: a report of the American College of Cardiology/ American Heart Association Task Force on Practice Guidelines (writing committee to revise the 1998 Guidelines for the Management of Patients With Valvular Heart Disease): developed in collaboration with the Society of Cardiovascular Anesthesiologists: endorsed by the Society for Cardiovascular Angiography and Interventions and the Society of Thoracic Surgeons. Circulation. 2006;114:e84-e231.

18. Quiñones MA, Otto CM, Stoddard M, et al. Recommendations for quantification of Doppler echocardiography: a report from the Doppler Quantification Task Force of the Nomenclature and Standards Committee of the American Society of Echocardiography. J Am Soc Echocardiogr. 2002;15:167-184.

19. Nanda NC, Roychoudhury D, Chung SM, Kim KS, Ostlund V, Klas B. Quantitative assessment of normal and stenotic aortic valve using transesophageal three-dimensional echocardiography. Echocardiography. 1994;11:617-625.

20. Menzel T, Mohr-Kahaly S, Kölsch B, et al. Quantitative assessment of aortic stenosis by three-dimensional echocardiography. J Am Soc Echocardiogr. 1997;10:215-223.

21. Ge S, Warner JG Jr, Abraham TP, et al. Three-dimensional surface area of the aortic valve orifice by three-dimensional echocardiography: clinical validation of a novel index for assessment of aortic stenosis. Am Heart J. 1998;136:1042-1050.

22. Kasprzak JD, Salustri A, Roelandt JR, Ten Cate FJ. Three-dimensional echocardiography of the aortic valve: feasibility, clinical potential, and limitations. Echocardiography. 1998;15:127-138.

23. Blot-Souletie N, Hébrard A, Acar P, Carrié D, Puel J. Comparison of accuracy of aortic valve area assessment in aortic stenosis by real time three-dimensional echocardiography in biplane mode versus two-dimensional transthoracic and transesophageal echocardiography. Echocardiography. 2007;24:1065-1072.

24. Goland S, Trento A, Iida K, et al. Assessment of aortic stenosis by three-dimensional echocardiography: an accurate and novel approach. Heart. 2007;93:801-807.

25. Gutiérrez-Chico JL, Zamorano JL, Prieto-Moriche E, et al. Real-time three-dimensional echocardiography in aortic stenosis: a novel, simple, and reliable method to improve accuracy in area calculation. Eur Heart J. 2008;29:1296-1306.

26. Poh KK, Levine RA, Solis J, et al. Assessing aortic valve area in aortic stenosis by continuity equation: a novel approach using real-time threedimensional echocardiography. Eur Heart J. 2008;29:2526-2535.

27. Doddamani S, Bello R, Friedman MA, et al. Demonstration of left ventricular outflow tract eccentricity by real time $3 \mathrm{D}$ echocardiography: implications for the determination of aortic valve area. Echocardiography. 2007;24:860-866.

28. Pérez de Isla L, Zamorano J, Pérez de la Yglesia R, et al. Quantification of aortic valve area using three-dimensional echocardiography. Rev Esp Cardiol. 2008;61:494-500. Spanish.

29. Khaw AV, von Bardeleben RS, Strasser C, et al. Direct measurement of left ventricular outflow tract by transthoracic real-time 3D-echocardiography increases accuracy in assessment of aortic valve stenosis. Int J Cardiol. 2009;136:64-71.

30. Jilaihawi H, Doctor N, Kashif M, et al. Aortic annular sizing for transcatheter aortic valve replacement using cross-sectional 3-dimensional transesophageal echocardiography. J Am Coll Cardiol. 2013;61: 908-916.

31. Pershad A, Stone D, Morris MF, Fang K, Gellert G. Aortic annulus measurement and relevance to successful transcatheter aortic valve replacement: a new technique using 3D TEE. J Interv Cardiol. 2013;26: 302-309. 
32. Smith LA, Dworakowski R, Bhan A, et al. Real-time three-dimensional transesophageal echocardiography adds value to transcatheter aortic valve implantation. J Am Soc Echocardiogr. 2013;26:359-369.

33. Smith LA, Monaghan MJ. Monitoring of procedures: peri-interventional echo assessment for transcatheter aortic valve implantation. Eur Heart $J$ Cardiovasc Imaging. 2013;14:840-850.

34. Kasel AM, Cassese S, Leber AW, von Scheidt W, Kastrati A. Fluoroscopy-guided aortic root imaging for TAVR: "follow the right cusp" rule. JACC Cardiovasc Imaging. 2013;6:274-275.

35. Tzikas A, Schultz CJ, Piazza N, et al. Assessment of the aortic annulus by multislice computed tomography, contrast aortography, and transthoracic echocardiography in patients referred for transcatheter aortic valve implantation. Catheter Cardiovasc Interv. 2011;77:868-875.

36. Leipsic J, Gurvitch R, Labounty TM, et al. Multidetector computed tomography in transcatheter aortic valve implantation. JACC Cardiovasc Imaging. 2011;4:416-429.

37. Little SH, Shah DJ, Mahmarian JJ. Multimodality noninvasive imaging for transcatheter aortic valve implantation: a primer. Methodist Debakey Cardiovasc J. 2012;8:29-37.

38. Moss RR, Ivens E, Pasupati S, et al. Role of echocardiography in percutaneous aortic valve implantation. JACC Cardiovasc Imaging. 2008;1:15-24.

39. Zamorano JL, Badano LP, Bruce C, et al. EAE/ASE recommendations for the use of echocardiography in new transcatheter interventions for valvular heart disease. J Am Soc Echocardiogr. 2011;24:937-965.

40. Achenbach S, Delgado V, Hausleiter J, Schoenhagen P, Min JK, Leipsic JA. SCCT expert consensus document on computed tomography imaging before transcatheter aortic valve implantation (TAVI)/ transcatheter aortic valve replacement (TAVR). J Cardiovasc Comput Tomogr. 2012;6:366-380.

41. Detaint D, Lepage L, Himbert D, et al. Determinants of significant paravalvular regurgitation after transcatheter aortic valve: implantation impact of device and annulus discongruence. JACC Cardiovasc Interv. 2009;2:821-827.

42. Gurvitch R, Webb JG, Yuan R, et al. Aortic annulus diameter determination by multidetector computed tomography: reproducibility, applicability, and implications for transcatheter aortic valve implantation. JACC Cardiovasc Interv. 2011;4:1235-1245.

43. Okuyama K, Jilaihawi H, Makkar RR. Leaflet length and left main coronary artery occlusion following transcatheter aortic valve replacement. Catheter Cardiovasc Interv. Epub June 14, 2013.
44. Genereux P, Webb JG, Svensson LG, et al. Vascular complications after transcatheter aortic valve replacement: insights from the PARTNER (Placement of AoRTic TraNscathetER Valve) trial. J Am Coll Cardiol. 2012;60:1043-1052.

45. American College of Radiology, Society of Cardiovascular Computed Tomography, Society for Cardiovascular Magnetic Resonance, et al. ACCF/ACR/SCCT/SCMR/ASNC/NASCI/SCAI/SIR 2006 appropriateness criteria for cardiac computed tomography and cardiac magnetic resonance imaging. A report of the American College of Cardiology Foundation Quality Strategic Directions Committee Appropriateness Criteria Working Group. J Am Coll Radiol. 2006;3:751-771.

46. American College of Cardiology Foundation Task Force on Expert Consensus. D, Hundley WG, Bluemke DA, et al. ACCF/ACR/AHA/ NASCI/SCMR 2010 expert consensus document on cardiovascular magnetic resonance: a report of the American College of Cardiology Foundation Task Force on Expert Consensus Documents. J Am Coll Cardiol. 2010;55:2614-2662.

47. Tops LF, Wood DA, Delgado V, et al. Noninvasive evaluation of the aortic root with multislice computed tomography implications for transcatheter aortic valve replacement. JACC Cardiovasc Imaging. 2008;1:321-330.

48. Jabbour A, Ismail TF, Moat N, et al. Multimodality imaging in transcatheter aortic valve implantation and post-procedural aortic regurgitation: comparison among cardiovascular magnetic resonance, cardiac computed tomography, and echocardiography. J Am Coll Cardiol. 2011;58:2165-2173.

49. Gurvitch R, Wood DA, Leipsic J, et al. Multislice computed tomography for prediction of optimal angiographic deployment projections during transcatheter aortic valve implantation. JACC Cardiovasc Interv. 2010;3: 1157-1165.

50. Tzikas A, Schultz C, Van Mieghem NM, de Jaegere PP, Serruys PW. Optimal projection estimation for transcatheter aortic valve implantation based on contrast-aortography: validation of a prototype software. Catheter Cardiovasc Interv. 2010;76:602-607.

51. Binder RK, Leipsic J, Wood D, et al. Prediction of optimal deployment projection for transcatheter aortic valve replacement: angiographic 3 -dimensional reconstruction of the aortic root versus multidetector computed tomography. Circ Cardiovasc Interv. 2012;5:247-252.

52. Maeda K, Kuratani T, Torikai K, Shimamura K, Sawa Y. Transcatheter aortic valve replacement using DynaCT. J Card Surg. 2012;27: $551-553$.
Research Reports in Clinical Cardiology

\section{Publish your work in this journal}

Research Reports in Clinical Cardiology is an international, peerreviewed, open access journal publishing original research, reports, editorials, reviews and commentaries on all areas of cardiology in the clinic and laboratory. The manuscript management system is completely online and includes a very quick and fair peer-review system.

\section{Dovepress}

Visit http://www.dovepress.com/testimonials.php to read real quotes from published authors. 